\title{
Tangence
}

\section{Colins, ou : le socialisme rationnel}

\section{Marc Angenot}

Numéro 57, mai 1998

Littérateurs atypiques et penseurs irréguliers

URI : https://id.erudit.org/iderudit/025972ar

DOI : https://doi.org/10.7202/025972ar

Aller au sommaire du numéro

Éditeur(s)

Tangence

ISSN

0226-9554 (imprimé)

1710-0305 (numérique)

Découvrir la revue

Citer ce document

Angenot, M. (1998). Colins, ou : le socialisme rationnel. Tangence, (57), 111-118. https://doi.org/10.7202/025972ar d'utilisation que vous pouvez consulter en ligne.

https://apropos.erudit.org/fr/usagers/politique-dutilisation/ 


\section{Colins, ou : le socialisme rationnel \\ Marc Angenot, Université McGill}

Jean-Guillaume César-Alexandre Hippolyte,

Baron de Colins de Ham - dit Colins

(1783-1859)

Les leçons d'un jésuite théiste

De Bruxelles à Waterloo

Brève rencontre à Charenton avec Donatien Alphonse François, ci-devant Marquis de Sade

Le gouvernement de l'institution passé aux fous:

un rapport sévère à Royer-Collard

1817: Colins à Philadelphie

Peut-on s'échapper de Sainte-Hélène en ballon?

À Cuba, Colins, médecin, propriétaire d'esclaves et latifundiaire

Le père de Carlos Collins y San Jorge, compagnon de Martí

Retour à Paris en 1830: le solitaire de Montrouge

"Je suis prolétaire; sans ma demi-solde, je mourrais de faim."

Le vieil étudiant de cinquante ans

et la découverte de la vérité absolue

"Avoir vécu pour ainsi dire seul savant au sein d'un

Charenton social."

"Et des Charentons il y en a des milliards. En époque d'ignorance

sociale, il y en a au moins un chez chaque individu. "

Ce que peut lire le promeneur au vieux Cimetière de Montrouge:

L'ORDRE MORAL C'EST: L'HARMONIE ÉTERNELLE ENTRE IA LIBERTTÉ

DES ACTIONS ET LA FATALITÉ DES ÉVÉNEMENTS

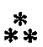

À quoi sert une histoire de l'oubli?

"Je mets ici ma signature devant la traite tirée par lui,

en toute confiance sur la postérité." (Émile de Girardin)

Les dix-neuf volumes de la Science sociale et les autres, et les quarante-quatre volumes d'inédits : une œuvre très inachevée Colins, glossateur total 
112

"Si écorcher un homme vif est peu amusant, écorcher vif l'esprit de toute une génération est moins amusant encore."

(Colins, De la justice, I 2)

"Trouver un public qui veuille me lire":

"cette condition sera seulement possible

lorsque l'anarchie l'aura rendue nécessaire "

(De la souveraineté, I 79).

"Je ne serai lu qu'après..."

Un auteur déplaisant: "Je publie cet ouvrage avec la certitude de déplaire à presque tout le monde. "(Du pacte social, 1835, préf.) Voir Joseph de Maistre, Les soirées de Saint-Pétersbourg, I :

"Il y a mille manières de se tromper,

il n'y en a qu'une d'avoir raison."

L'école des logocrates ou socialistes-rationnels

Grands récits et petites sectes, autres exemples:

les saint-simoniens, l'École sociétaire, le Familistère,

l'Apostolat positiviste...

Les grands disciples: Adolphe Hugentobler, Louis \& Agathon de

Potter, Jules Putsage, Frédéric Borde...

Le culte de Colins et la vulgarisation de sa Science sociale

Fidélité des colinsiens, schismes des positivistes et censures des fouriéristes

"Je meurs dans la religion rationnelle. "(Louis de Potter)

Passage à l'acte: les colinsiens au Costa-Rica, Escuela Colins

Le "collectivisme belge, ou : Colins, prophète en son pays"

Les colinsiens français

La philosophie de l'avenir et La société nouvelle

Ce qui est mort le deux août 1914

***

La déconstruction sous Louis-Philippe

L'Utopie comme envers de la critique radicale:

Pars construens $\mathcal{E}$ Pars destruens

Une pensée griffonnée en marge de tous les livres

Comment lire synoptiquement Bonald, de Maistre, Say, Sismondi,

Fourier, Considérant, Cousin et Proudhon?

Apories et antinomies

Le spiritualisme et le matérialisme

comme auto-réfutations réciproques:

Qui s'appuie sur l'un démontre l'autre - et vice-versa

La vertu moderne: "d'après $M$. Jules Simon, un acte n'est vertueux 
que si, méritant une récompense, elle ne lui est pas accordée." Colins et Proudhon - dans ses contradictions, celui-ci, De la justice: "Si l'homme était tout matière, il ne serait pas libre. $\mathrm{Ni}$ l'attraction, ni aucune combinaison des différentes qualités des corps ne suffit à constituer le libre-arbitre: le sens commun suffit

à le faire comprendre. "Mais du même, dans Système des contradictions : "nous sommes... des ressorts pensants, des roues pensantes, des pignons pensants, des poids pensants, etc. d'une

immense machine qui pense aussi et qui va toute seule."

L'imprimerie et le développement de l'examen

Sans fin ni cesse: "Il est impossible de poser des bornes à l'esprit d'examen, de lui dire: tu n'iras pas au-delà de tel point. " ( $L a$ philosophie de l'avenir, octobre 1889, 79)

L'“incompressibilité de l'examen" ou l'anarchie moderne

"La liberté de conscience, c'est-à-dire l'ignorance, mise en présence de l'incompressibilité de l'examen, est précisément ce qui constitue: l'époque anarchique dans laquelle nous nous trouvons. " (Colins, De la souveraineté, I 21)

Comment surmonter l'ignorance présente quant à la réalité du droit?

Plus d'autorité religieuse -

pas encore de souveraineté de la raison:

Interrègne et anarchie.

La "souveraineté du peuple" comme souveraineté de l'ignorance: "On en est réduit pour déterminer ce que l'on convient à chaque époque d'appeler vérité et justice, à consulter les membres délibérants de la société et à s'arrêter à la décision que prend la moitié des votants plus un." (Louis de Potter, in Études sociales, 11)

"Cette opinion [publique], c'est le masque de raison sous lequel se cache la force qui domine la société..." (Louis de Potter, De la réalité, vi)

Qu'il n'y a que deux façons de comprimer l'examen : $1=3$ ou $1+1=2$

Epistémè ou topographie des antinomiques?

Ungleichzeitigkeit (Ernst Bloch):

non-contemporanéité des contemporains

Matérialisme et libre arbitre

L'homme, le chien et la carotte, ou le sophisme de la

"Série continue des êtres"

Liberté réelle et justice éternelle

"Si lé Dieu personnel existe; lui seul est un MOI réel; et, tous les autres moi ne sont: que des apparences..." 
114

"Si Dieu existe, il n'y a ni liberté, ni vice, ni vertu." (mais s'il n'existe pas, il n'y en a pas non plus!)

Le jeu de la double erreur et du tiers exclu 10.000 ans d'une erreur n'en font pas une vérité, disait Brecht:

"Depuis le commencement du monde l'anthropomorphisme existe et cependant l'anthropomorphisme est évidemment une erreur." (Colins, Science sociale, I 58)

Le matérialisme ne se soutient que par ses inconséquences:

Français encore un effort si vous voulez être matérialistes!

Colins, ou l'immatérialisme historique

De la réalité déterminée par le raisonnement

$\mathrm{Ni}$ de votre Église, ni de votre Révolution

L'ordre social entièrement démontré par l'absurde "Aucune souveraineté ne peut être ouvertement basée sur une

force séparée de toute espèce de raisonnement, de justice; l'impossibilité d'établir la souveraineté sur le raisonnement réel, incontestable, oblige à établir des souverainetés sur des raisonnements illusoires, contestables."

L'épistémologie de Sherlock Holmes, ou la preuve par l'inacceptable: "En époque d'ignorance, il n'est qu'une seule

chose qui ne puisse être admise: la vérité."

(Colins, La pbilosophie de l'avenir, 1880, 161)

Critère épistémique: Avoir raison, c'est être pris pour fou: "Galilée et moi"

Que l'Âge théocratique a d'ailleurs parfaitement bien fait de condamner Socrate et Galilée

Science unique et idéologies multiples: "Le socialisme rationnel est la science exacte par excellence car son point de départ: je me sens exister, est absolument inattaquable." (La philosophie de l'avenir, août 1892, 14)

Cherche désespérément la science réelle "sous peine de mort sociale"

"On ne peut faire un plébiscite sur l'unité mathématique."

Les lois se découvrent, elles ne se votent pas

La religion rationnelle sera démontrée

Penser le présent comme scandale

L' "enfer social" et le monde comme ce qui "ne peut plus durer"

"Cette anarchie ne fait que commencer..."

"The One Who Walked Away From Omelas" (une nouvelle d'Ursula K. LeGuin)

Fichte et l'ère de la culpabilité universelle

Tirer une preuve du scandale:

fondation de toute rhétorique sur l'improbable 
Le sorite du mal social

"La société actuelle est malade; le mal social actuel est l'anarchie;

la cause du mal social est l'ignorance relative: à la réalité du droit..." (Colins, inédit, Revue du socialisme rationnel, vol. 1903, 564)

Le bien qui se transforme en mal: "Aujourd'hui tout progrès dans le système de la civilisation entraîne un péjoratif; toute prospérité entraîne une extension du cancer social; et notre organisation industrielle est une machine immense qui fait des pauvres et des

prolétaires. " (Victor Considérant, cité par Colins,

De la souveraineté, II 569)

Le mal social et son remède

L'"Extinction du paupérisme", de l'oncle au neveu

Paupérisme et ignorance, ou paupérisme moral

Y a-t-il des préconditions morales au socialisme?

À quelle condition l'honnête homme n'est-il pas

un pur et simple jobard?

Scandale actuel et Grands récits

Une démonstration "socialement irréfutable", ou :

l'art de différer ses preuves

Martin Heidegger dans Sein und Zeit: comment parler de l'être?

I. Si l'homme est libre, Dieu n'existe pas

II. Puisque Dieu n'existe pas, l'âme existe - éternellement

Démonstration obscure de la "sanction ultravitale"

Voyez aussi bien les fouriéristes pour comprendre "le XIX ${ }^{e}$ siècle à

travers les âges" (Philippe Muray): "Ainsi dans la série d'existences que nous parcourons,

nous avons derrière nous un passé et devant nous un avenir...

Nous faisons un voyage à travers les siècles et à travers les

astres... ALTERNAT éternel de sexe à sexe, de caractère à

caractère, de planète à planète." (Jules Lechevalier, Études, 37)

L'âme et l'oubli : une métempsycose sans anamnèse

Les animaux-machines et la sensibilité réelle

L'âme comme logos: "une langue sous quelque forme que ce soit est un raisonnement "(Colins, Science sociale, III 349)

La Primauté du verbe, indice de la sensibilité réelle

Colins, produit du condillacisme -

et de De Gérando et autres idéologues

Un texte sur l'évolution - dix ans avant The Origin of Species: "La transformation successive des espèces est irrécusable. Goethe doit être placé à la tête des philosophes pour l'avoir reconnu..."

(Science sociale, I 93) 
Mais : "Le singe est-il intelligent? Eh, s'il l'est, c'est un être moral, c'est votre frère. S'il l'est, il a autant que vous droit à l'égalité devant la loi." (Ibid., I 98) "c'est à cet arbitraire que l'on doit de placer le boschimane au rang des singes". (ibid., I 166)

Lisez Clémence Royer, Origine de l'bomme, 171, si vous préférez la science établie et respectée: "un Papou est plus proche non seulement du singe, mais du kangourou, que d'un Descartes ou d'un Newton."

Colins ou l'antiracisme

Ce que Marx avait compris de Colins: "[si les animaux avaient des sentiments,] nous serions des cannibales et l'on ne pourrait jamais fonder un empire de justice sur terre" (Lettre à Sorg, -

citée avec mépris dans Philosophie de l'avenir, vol. 1892)

La vie humaine et ses souffrances comme expiation, "la conséquence rationnellement nécessaire des fautes commises contre la raison dans une vie précédente (Louis De Potter,

Dictionnaire rationnel, 131)

Colins et Ballanche, La ville des expiations

L'، harmonie éternelle entre la liberté des actions et la fatalité des événements."

"Toute humanité se divise nécessairement en trois époques": Les trois stades historiques, théocratie, démocratie, logocratie

Progrès et catastrophe

De l'ignorance primitive au socialisme scientifique

Dialectique de la liberté et de la soumission à la science: "Quand forcé par l'évidence de la démonstration, on dit que deux et deux font quatre en est-on moins libre? Sous la souveraineté de la force brutale, sous la souveraineté personnelle, les sujets sont esclaves. Sous la souveraineté impersonnelle de la

raison incontestable, ils seront libres. "(Agathon de Potter, Economie sociale, I 11)

Comparaison avec les autres systèmes totaux, où repérer les Sujets de l'histoire: Saint-Simon, Colins, Comte, Marx \& les autres

Colins et Marx, Ungleichzeitigkeit, le contraire et le même

Colinsiens vs Marxistes: "Pour les curés de la sociale, les capitalistes ne sont pas aussi clairvoyants que les hiérophantes du

collectivisme. Ils sont tellement pénétrés de la puissance messianique du mythe libérateur que les marxistes impénitents nous assurent avec un sérieux qui déconcerte que les capitalistes creusent de leurs propres mains la tombe où ils doivent être ensevelis." (C. Soubeyran, Revue du socialisme rationnel, août 1911, 44) 
Se demander si le Capital ne suggère pas la perpétuation indéfinie du capitalisme, admis que le Manifeste communiste prophétise la Révolution et que la Critique du programme de Gotha organise l'État socialiste...

Le matérialiste et ses pauvres

Abolir le paupérisme moral

L'inventeur du mot "collectivisme"

L'aliénation du sol et l'abolition de l'héritage collatéral "La souveraineté du peuple, ou la démocratie, ou le régime bourgeois enfin, engendre NÉCESSAIREMENT l'appropriation individuelle du sol et par conséquent un paupérisme croissant proportionnellement au développement de la richesse générale."

(Agathon de Potter, Économie sociale, I 9)

Un collectivisme limité à l'appropriation du sol

Colins et Henry George, le socialisme agraire La Société future

Les logocrates, membres du Souverain

Perspective catastrophiste, une histoire sans sujet

Une alternance indéfinie d'anarchie et de despotisme

Phase de transition et dictature - Colins, Comte et Marx Une dictature sur le prolétariat; le Logocrate, " un autocrate... unissant la force à la raison... pour détruire l'ignorance sociale" "Si vous avez un Charenton à guérir, il faut commencer par être le plus fort. Sinon, le gouvernement passe aux fous. Et c'est peu amusant. Pour guérir un Charenton, il faut donc avoir: des garde-

fous, des camisoles de force, des douches, des triques, des

knouts..." (Colins, Science sociale, V, 462-3)

Ce que sera le règne de la science et la fin de l'histoire:

"L'Ordre social est devenu imperturbable et il dure jusqu'à la mort de l'humanité sur le globe." (Agathon de Potter, Revue du socialisme rationnel, 1903, 514)

Unité sociale de l'humanité : "fusion de l'humanité en une seule classe, celle des travailleurs, gagnant en proportion de leur travail."

(Bufquin des Essarts, Socialisme..., 12)

"L'idée que l'humanité puisse cesser d'être divisée en sociétés distinctes fait sourire de pitié les hommes à courte vue."

(Louis de Potter, Dictionnaire rationnel, 292)

Socialisme rationnel ou barbarie: "la vérité doit être socialement reconnue ou la société doit retourner à la sauvagerie"

(Jules Putsage, Nécessités sociales, 10)

Les Grands récits contre la démocratie 
Mably et Morelly, le totalitarisme des Lumières

Ordre et Progrès, ou : Le drapeau brésilien

***

Y a-t-il une épistémè des Grands récits?

Connaissance historique et Propagande militante

L'histoire-maëlstrom, ou : Les aventures d'un certain Hans Pfall

Conjurer la déréliction et le désenchantement

Pourquoi vouloir faire le bonheur des hommes?

Mener l'histoire à bonne fin

Fatalité sans agent historique: "Du mal être de la plupart naissent les révolutions; du désespoir de tous naîtra la rénovation sociale; elle ne sera faite par personne, elle se fera." (Louis de Potter, Etudes sociales, I iv)

Grands récits, récits de soi, et modernité

Une "quête démonique de valeurs authentiques menée par un héros problématique dans une société dégradée "(Lukács) Colins est dans le $\mathrm{XTX}^{\mathrm{e}}$ siècle comme un poisson dans l'eau Le marché des "solutions sociales" au tournant du siècle: Abolitionnistes, pacifistes, espérantistes, féministes, naturiens, néo-malthusiens, théosophes, végétariens \& les autres

Le mouvement ouvrier, socialiste, révolutionnaire

Un siècle-charnière, - suivi d'un siècle-charnier Fin de la modernité 